\title{
Dynamic Modeling of a Spatial Cable-Driven Continuum Robot Using Euler-Lagrange Method
}

\author{
Ammar Amouri ${ }^{1, *}$, Chawki Mahfoudi ${ }^{2}$, Abdelouahab Zaatri $^{3}$ \\ ${ }^{1}$ Department of Mechanical Engineering, Faculty of Technology Sciences, University of Brothers Mentouri, \\ Constantine 1, Algeria \\ ${ }^{2}$ Department of Mechanical Engineering, University Labri Ben M'Hidi, Oum el Bouaghi, Algeria \\ ${ }^{3}$ Independent Researcher, Ex-Department of Mechanical Engineering, University of Brothers Mentouri, \\ Constantine 1, Algeria
}

Received 10 June 2019; received in revised form 29 August 2019; accepted 08 October 2019

DOI: https://doi.org/10.46604/ijeti.2020.4422

\begin{abstract}
Continuum robots are kinematically redundant and their dynamic models are highly nonlinear. This study aims to overcome this difficulty by presenting a more practical dynamic model of a certain class of continuum robots called cable-driven continuum robot (CDCR). Firstly, the structural design of a CDCR with two rotational degrees of freedom (DOF) is introduced. Then, the kinematic models are derived according to the constant curvature assumption. Considering the complexity of the kinetic energy expression, it has been approximated by the well-known Taylor expansions. This case corresponds to weak bending angles within the specified bending angle range of the robot. On the other hand, due to the low weight of the CDCR components, the gravitational energy effects can be neglected compared to those stemmed from the elastic energy. Thereafter, the corresponding dynamic model is established using Euler-Lagrange method. Static and dynamic models have been illustrated by examples. This analysis and dynamic model development have been compared with the existing scientific literature. The obtained results shown that the consistency and the efficiency of accuracy for real-time have been carried out. However, the dynamic modeling of CDCR with more than 2-DOF leads to a more complex mathematical expression, and cannot be simplified by adopting the similar assumptions and methodology used in the case of 2-DOF.
\end{abstract}

Keywords: continuum robot, cable-driven continuum robot, Taylor expansions approximations, Euler-Lagrange method, dynamic modeling

\section{Introduction}

Continuum robots are behavioral-extension of hyper redundant manipulators inspired by animal world, even vegetable [1]. They can be made of hard or soft structures [2-3]. Unlike most conventional robots, continuum robots are characterized by continuous and jointless flexible backbone. The motion of the robot is controlled by the mechanical actuators along with the structure through the cables, tendons, wires or pneumatic/hydraulic bellows. This category of hyper redundant manipulators is conceived to mimic the extraordinary capacities of some remarkable animal structures such as elephant trunks, tentacles and snakes, which can offer a wide capacity range of maneuverability, dexterous manipulation and cluttered environments [4-10].

Unlike conventional robots, the forward kinematic modeling of continuum robots is more difficult to be modeled, and that is due to their structural complexity, the strong coupling of their operational variables, and theoretical possibility of having an infinite number of DOF. However, these continuum robots can be controlled only by means of a finite number of DOF, which

* Corresponding author. E-mail address: ammar_amouri@yahoo.fr 
reduces the set of solutions. In this paper, many research works have been proposed in literature to accomplish this purpose [11-18].

Regarding dynamics modeling of continuum robots from a general point of view, there are many research works that have been proposed in the literature. These models have been derived by different methods for single and multi-bending sections, and for both planar and spatial manner cases such as, Euler-Lagrange formalism [15, 19], Cosserat rod theory [20-22], Newton-Euler formalism [23], Hamilton principle [24] and virtual power principle [25-26].

For the considered class of continuum robots called cable-driven continuum robots (CDCRs), there are a few works about the dynamic modeling [15, 24-26]. He et al. [15] presented the dynamic model of a specified continuum robot with one bending section. The model was derived using the Lagrange method based on the Bernoulli beam equation and with the constant curvature assumption. Gravagne et al. [24] presented the dynamic model of a planar cable-driven robot using the Hamiltons principle based on an appropriate planar large deflection. Rone and Ben-Tzvi [25] described the dynamic models of a single section for a cable-driven continuum manipulator using the principle of virtual power taking into consideration inertial, frictional and gravitational effects. The same authors extended this model of a multi-segment rod driven continuum robot by considering the existence of friction between rods and disks [26]. However, these models are numerically complex and very difficult to implement for control purposes or for being used in general three-dimensional case.

In our previous work [27], the planar dynamic model of CDCR was developed on the basis of geometric model approximation by Taylor expansions with fixed orientation assumption (i.e. planar projection). Although no inclusion of many effect terms (such as the frictional and gravitational effects) in the developed model. The obtained simulation results show some similarity for static and dynamic responses of the continuum robot behavior compared to works available in literature [15, 25]. This study is extended to the three-dimensional case (3D) with, just for one bending section.

However, since the complexity of the analysis increases with the consideration of the third dimension compared to our two dimensions previous work approach [27], some simplifications were necessary to facilitate the development of the 3D dynamic model conception.

In this paper, the establishment of the kinematic models and their corresponding dynamic model leads to complex expressions which are difficult to analyze and exploit due to non linearity that appears in kinematic energy expressions. For these reasons, some expressions of the kinematic energy are approximated through the well-known Taylor expansions under the assumption of weak bending angle, which yields a simplified dynamical model and thus overcomes some numerical singularities of the kinematic model expressions. As a result, the simplified dynamic model becomes more exploitable and can thus be easily used for control purposes because of its reduced expressions [27-28]. For validation tests, the simplified dynamic model has been used to simulate the static equilibrium as well as the dynamic behavior of 2-DOF CDCR, whereas the inverse dynamic model has been used to simulate spatial trajectories tracking.

The remaining sections of this paper are organized as follows. Section 2 describes the structural design of 2-DOF CDCR In Section 3, the kinematic models are derived under constant curvature assumption and without torsion. In Section 4, the dynamic model is developed by using Euler-Lagrange method and on the basis of simplified expressions obtained by applying Taylor expansions approximations. The dynamic models are validated by simulation examples in Section 5. Conclusion and future works are given at the end.

\section{Cable-Driven Continuum Robot}

The explicative scheme of 2-DOF CDCR is shown in Fig. 1. The overall structural is composed of four basic components: The rigid base which comports the control system, flexible backbone which represents the principal element of the continuum robot, a number of disks and three actuation cables. 
Through independent controlling two cables, two degrees of freedom can be achieved. The first rotation is the bending angle $\theta$ and the second one is the orientation angle $\varphi$ (see right side of Fig. 1). Therefore, the spatial motion e.g. the deflection of the flexible backbone is governed by applying adequate tension to one or two cables, at the same time, placed at $120^{\circ}$ from each other.
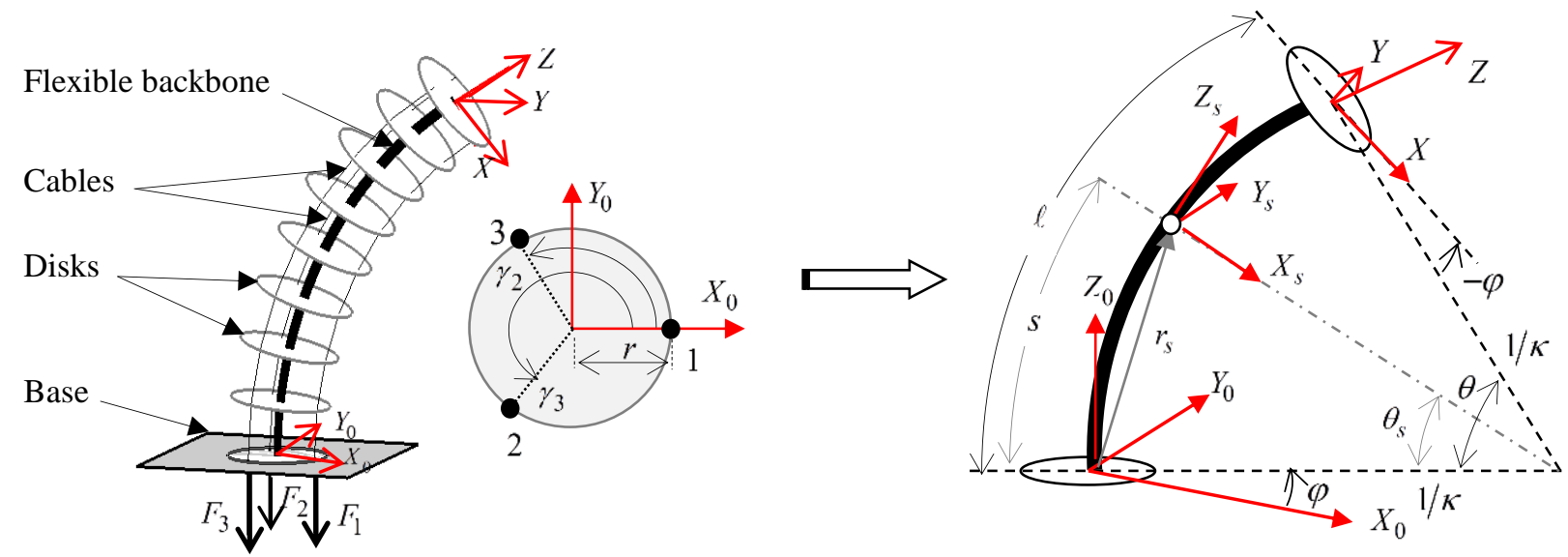

Fig. 1 Explicative scheme of the 2-DOF CDCR and its kinematics nomenclature

With respect to the underlined goal, the profile of the whole robot is assimilated to a backbone curve. This backbone curve is modeled as an inextensible arc of circle oriented in space, and parameterized by its arc length $\ell$, a curvature $\kappa$ and an orientation angle $\varphi$.

To describe the 2-DOF CDCR under consideration, three reference frames are defined: the first one is attached to the fixed disk $\left\{X_{0}, Y_{0}, Z_{0}\right\}$. The second frame $\{X, Y, Z\}$ is attributed to the end disk, and the last one is the moving frame $\left\{X_{s}, Y_{s}, Z_{s}\right\}$ that depends on the curve parameter $s \in[0, \ell]$.

\section{Kinematic Modeling}

This section defines the kinematic models of the 2-DOF CDCR. The modeling assumptions used in this paper are introduced at first. Then, positions, orientations and velocities are derived.

\subsection{Modeling assumptions}

The developments of kinematic and dynamic models are based on the following assumptions [27]:

- The flexible backbone is considered to be inextensible, and its shape is assumed to be continuous with constant curvature and zero torsion;

- The friction at each disk's cable routing hole are neglected;

- No external forces, except driving forces;

- The flexible backbone has a uniform mass distribution along its length, and possesses a high stiffness that permits the torsion avoiding.

\subsection{Position and orientation}

Each point located on the central axis of the flexible backbone is specified with $s$, which represents the length from the origin of reference frame to the specified point (see right side of Fig. 1).

Therefore, based on the constant curvature assumption [18], the position vector $\mathrm{r}_{s}$ of any point at $s$ can be expressed as 


$$
\mathrm{r}_{s}=\left[\begin{array}{lll}
\frac{s}{\theta_{s}}\left(1-\mathrm{c}\left(\theta_{s}\right)\right) \mathrm{c}(\varphi) & \frac{s}{\theta_{s}}\left(1-\mathrm{c}\left(\theta_{s}\right)\right) \mathrm{s}(\varphi) & \frac{s}{\theta_{s}} \mathrm{~s}\left(\theta_{s}\right)
\end{array}\right]^{T}
$$

where $\theta_{s}=\frac{s}{\ell} \theta$, and with abbreviations: $\mathrm{c} \equiv \cos ($.$) and \mathrm{s} \equiv \sin ($.$) .$

The orientation matrix can be defined by three sequential rotation matrices as follows:

$$
\mathrm{R}_{s}=\operatorname{rot}\left(Z_{0}, \varphi\right) \cdot \operatorname{rot}\left(Y_{0}, \theta_{s}\right) \cdot \operatorname{rot}\left(Z_{0},-\varphi\right)=\left[\begin{array}{lll}
\mathrm{n}_{s} & \mathrm{~b}_{s} & \mathrm{t}_{s}
\end{array}\right]
$$

where $\mathrm{n}_{s}, \mathrm{~b}_{s}$ and $\mathrm{t}_{s}$ are the normal vector, the binormal vector and the tangent vector correspond, respectively, to the first, second and third column of the matrix $\mathrm{R}_{s}$.

\subsection{Linear and angular velocities}

The linear velocity $\mathrm{v}_{s}$ at any point can be obtained by direct derivation, with respect to time, of Eq. (1). Whereas, the angular velocity $\omega_{s}$ can be expressed as [25]

$$
\omega_{s}=\widehat{\mathrm{t}}_{s} \cdot \dot{\mathrm{t}}_{S}
$$

where $\mathrm{t}_{s}$ is the derivation vector with respect to time, and $\hat{\mathrm{t}}_{s}$ is the skew matrix associated with vector $\mathrm{t}_{s}$ given by the third column of matrix $\mathrm{R}_{s}$ as

$$
\mathrm{t}_{s}=\left[\begin{array}{lll}
\mathrm{c} \varphi \mathrm{s} \theta_{s} & \mathrm{~s} \varphi \mathrm{s} \theta_{s} & \mathrm{c} \theta_{s}
\end{array}\right]^{T}
$$

\section{Dynamic Modeling}

To derive the equations of motion, Euler-Lagrange method is employed for the general coordinates $q=\left[\begin{array}{ll}\theta & \varphi\end{array}\right]^{\mathrm{T}}$ as follows:

$$
\frac{d}{d t} \frac{\partial \mathrm{T}}{\partial \dot{q}}-\frac{\partial \mathrm{T}}{\partial q}+\frac{\partial \mathrm{U}}{\partial q}=\mathrm{Q}_{j}
$$

where $\mathrm{T}$ is the total kinetic energy, $\mathrm{U}$ is the total potential energy, and $\mathrm{Q}_{j}$ is the generalized forces with $j=1,2$.

\subsection{Total kinetic energy}

The total kinetic energy of the 2-DOF CDCR consists of two parts: the kinetic energy of the flexible backbone $T_{b}$ and the kinetic energy of all disks $T_{d}$. Thus, the total kinetic energy is given as follows:

$$
\mathrm{T}=T_{b}+T_{d}
$$

where

$$
\begin{aligned}
& T_{b}=T_{b, \text { Trans }}+T_{b, \text { Rot }} \\
& T_{d}=T_{d, \text { Trans }}+T_{d, \text { Rot }}
\end{aligned}
$$

\subsubsection{Translational kinetic energy of flexible backbone}

The distribution mass of flexible backbone is assumed uniform; thus, the translational kinetic energy can be obtained as 


$$
T_{b, \text { Trans }}=\frac{1}{2} \int_{0}^{\ell} \mathrm{v}_{s}^{T} \mathrm{~m}_{b} \mathrm{v}_{s} d s=\frac{1}{2} \ell^{2} \mathrm{~m}_{b}\left(\frac{1}{3} H_{1} \dot{\theta}^{2}+\frac{1}{4} H_{2} \dot{\varphi}^{2}\right)
$$

where $\mathrm{m}_{b}$ is the flexible backbone mass, and factors $H_{1}$ and $H_{2}$ are given as follows:

$$
\begin{aligned}
& H_{1}=\frac{1}{\theta^{5}}\left(\theta^{3}+6 \theta-12 \mathrm{~s}(\theta)+6 \theta \mathrm{c}(\theta)\right) \\
& H_{2}=\frac{1}{\theta^{3}}(6 \theta-8 \mathrm{~s}(\theta)+\mathrm{s}(2 \theta))
\end{aligned}
$$

In the following, Taylor expansions are implemented in order to reduce the complexity of expressions that will be involved in the dynamic model's terms. They also serve to avoid the numerical singularities when $\theta$ is close to zero [27-28], which are clearly shown in expressions of Eq. (1) and the ones provided by its derivation as the expressions of factors $H_{i}$. Thus, by using the Taylor expansion with respect to the bending angle $\theta$, the equivalent factors of $H_{1}$ and $H_{2}$ are given as follows:

$$
\begin{aligned}
& \bar{H}_{1}=\frac{\theta^{4}}{8640}-\frac{\theta^{2}}{168}+\frac{3}{20} \\
& \bar{H}_{2}=-\frac{\theta^{4}}{42}+\frac{\theta^{2}}{5}
\end{aligned}
$$

CDCRs are constrained to a small range of the bending angle $\theta$ depending on the flexible backbone material. Therefore, for the range of the bending angle as $\theta \in\left[\begin{array}{ll}-3 \pi / 5 & 3 \pi / 5\end{array}\right]$, the Taylor expansions can approximate the factors involved in Eq. (9) with smaller errors, which are shown in Fig. 2.
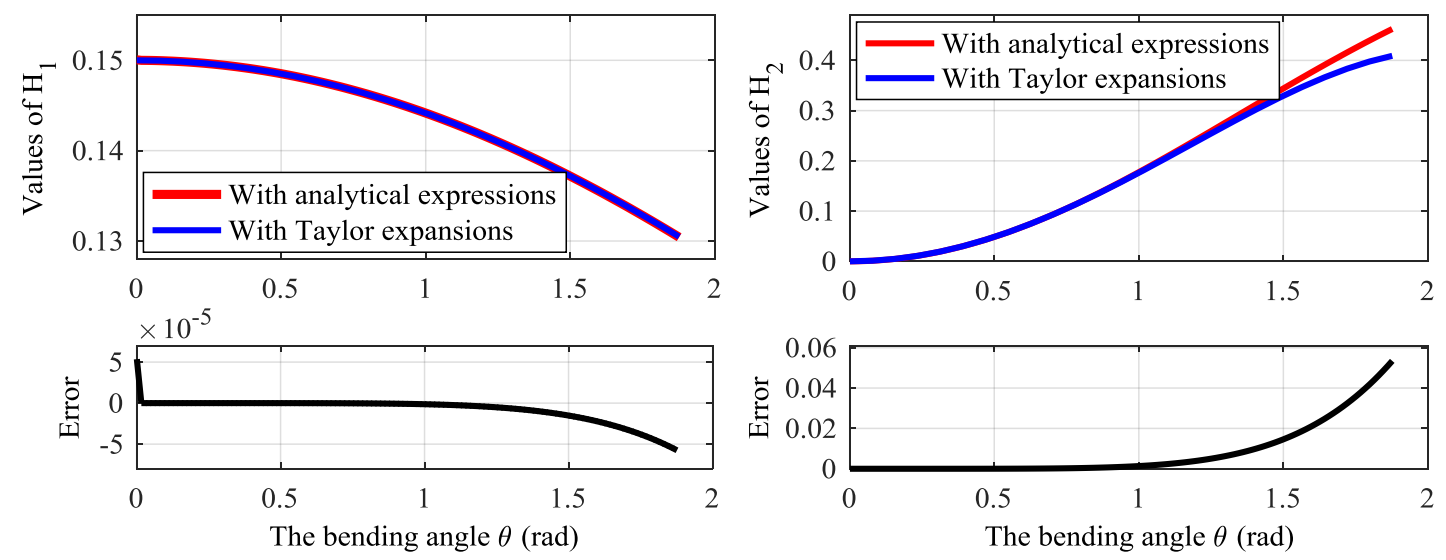

Fig. 2 The comparison of exact and equivalent factors of $H_{1}$ and $H_{2}$, and their errors

\subsubsection{Rotational kinetic energy of flexible backbone}

Without loss of generality, the rotational kinetic energy can be expressed as

$$
T_{b, R o t}=\frac{1}{2} \int_{0}^{\ell} \omega_{s}^{T} \mathrm{I}_{b} \omega_{s} d s=\frac{1}{2} \ell \mathrm{I}_{b}\left(H_{3} \dot{\theta}^{2}+H_{4} \dot{\varphi}^{2}\right)
$$

where $\mathrm{I}_{b}$ is the second moment of cross-sectional area of the flexible backbone, and the factors $H_{3}$ and $H_{4}$ are defined in the appendix. Similarly, the comparison of exact and equivalent factors of $H_{3}$ and $H_{4}$ in the specified range of the bending angle are shown in Fig. 3. 

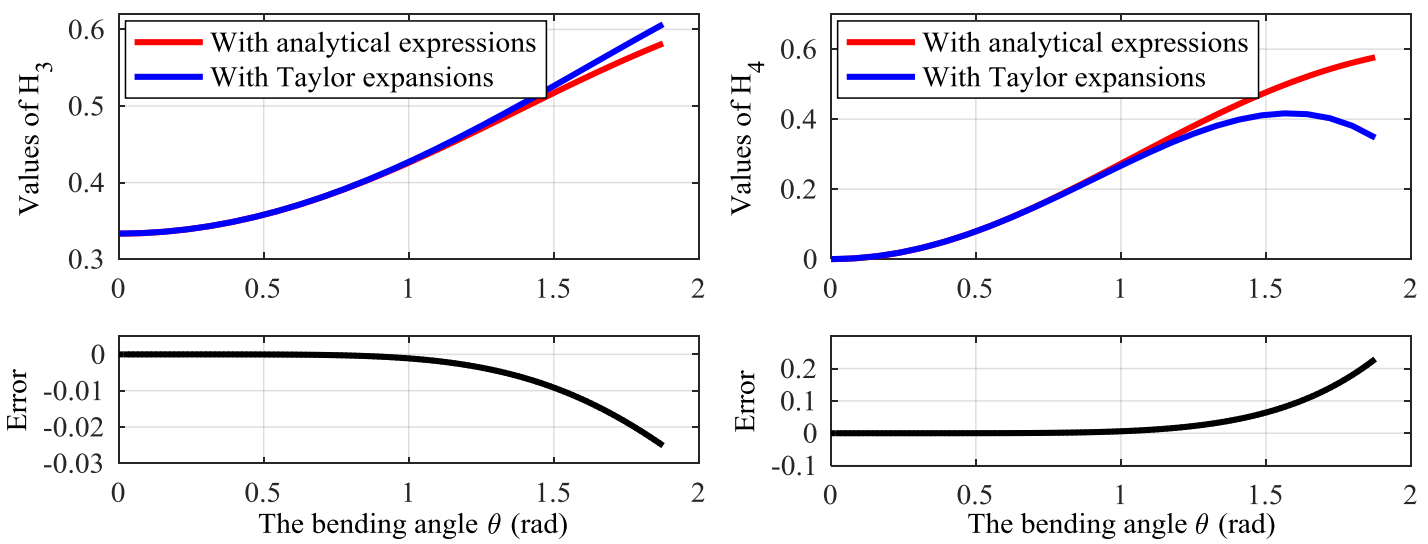

Fig. 3The comparison of exact and equivalent factors of $H_{3}$ and $H_{4}$, and their errors

\subsubsection{Translational kinetic energy of all disks}

For reasons of simplicity, the disks are mounted on the flexible backbone with equal space between each disk; therefore, the kinetic energy can be expressed as

$$
T_{d, \text { Trans }}=\frac{1}{2} \sum_{k=1}^{10} \mathrm{v}_{k}^{T} \mathrm{~m}_{d} \mathrm{v}_{k}=\frac{1}{2} \ell^{2} \mathrm{~m}_{d}\left(H_{5} \dot{\theta}^{2}+H_{6} \dot{\varphi}^{2}\right)
$$

where $\mathrm{m}_{d}$ is the disk's mass, and for each disk $k$, the linear velocity $\mathrm{v}_{k}$ is calculated at $s=k \ell / 10$ with $k=1,2, \ldots, 10$, and factors $H_{5}$ and $H_{6}$ are defined in the appendix. Fig. 4 shows the comparison of exact and equivalent factors of $H_{5}$ and $H_{6}$
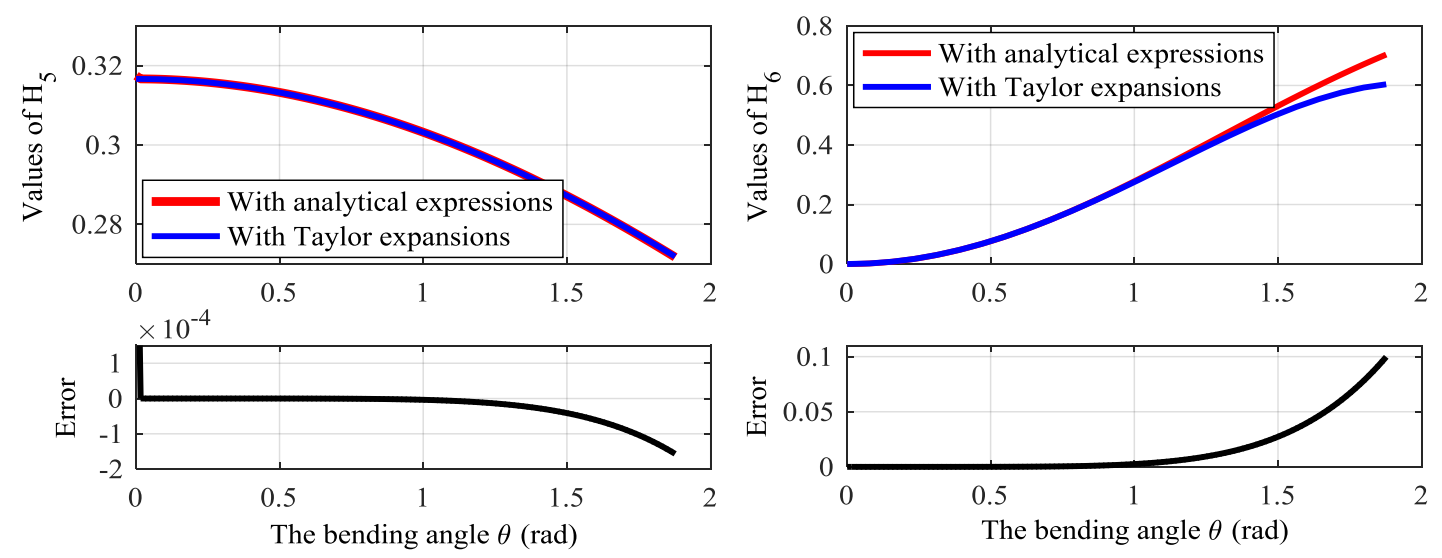

Fig. 4 The comparison of exact and equivalent factors of $H_{5}$ and $H_{6}$, and their errors

\subsubsection{Rotational kinetic energy of all disks}

Similarly to translational kinetic energy, the rotational energy can be written as

$$
T_{d, R o t}=\frac{1}{2} \sum_{k=1}^{10} \omega_{k}^{T} \mathrm{I} \omega_{k}=\frac{1}{2} \mathrm{I}_{x x}\left(H_{7} \dot{\theta}^{2}+H_{8} \dot{\varphi}^{2}\right)
$$

where $\mathrm{I}$ is the disk's moment of inertia expressed in the reference frame $\left\{X_{0}, Y_{0}, Z_{0}\right\}$ which depends on the disk's orientation and local moments of inertia $\mathrm{I}_{x x}, \mathrm{I}_{y y}$, and $\mathrm{I}_{z z}$. This moment of inertia can be expressed as

$$
\mathrm{I}={ }^{0} \mathrm{R}_{k}\left(\begin{array}{ccc}
\mathrm{I}_{x x} & 0 & 0 \\
0 & \mathrm{I}_{y y} & 0 \\
0 & 0 & \mathrm{I}_{z z}
\end{array}\right){ }^{0} \mathrm{R}_{k}^{T}
$$


and factors $H_{7}$ and $H_{8}$ are defined in the appendix. The comparison of exact and equivalent factors is shown in Fig. 5.
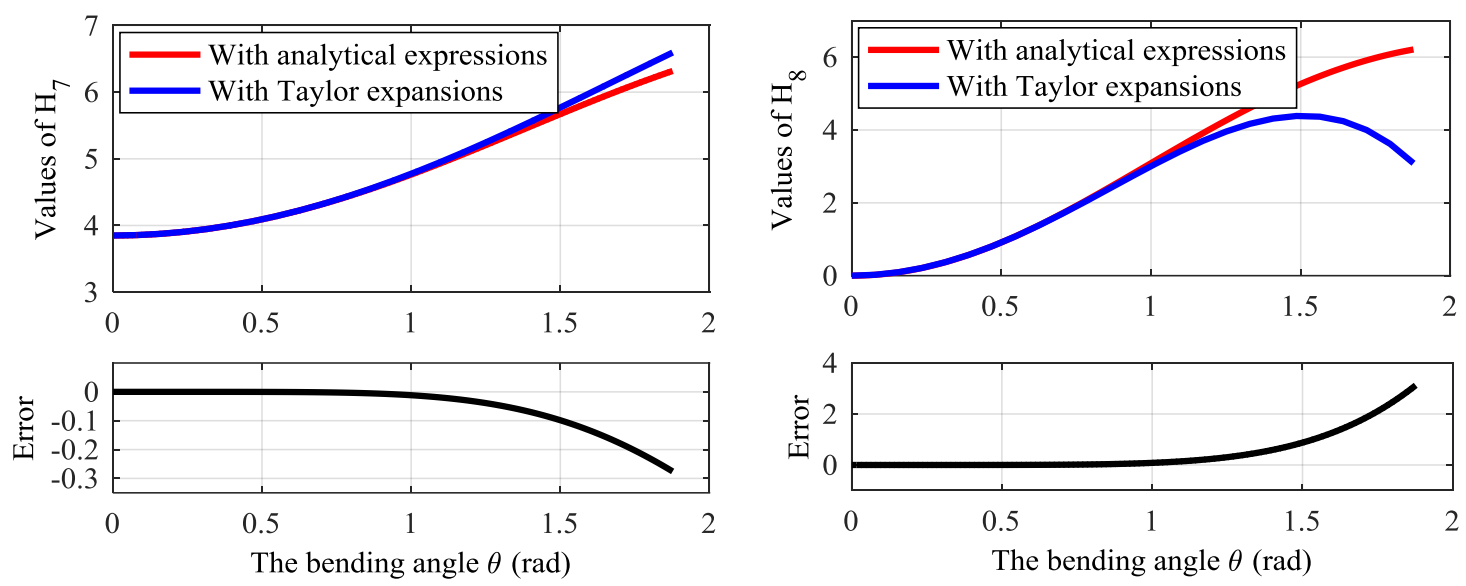

Fig. 5 The comparison of exact and equivalent factors of $H_{7}$ and $H_{8}$, and their errors

Table 1 The estimated parameters and geometric properties of 2-DOF CDCR

\begin{tabular}{ccc}
\hline \hline Parameters & Designation & Value \\
\hline$\ell$ & Length of flexible backbone & $0.802 \mathrm{~m}$ \\
$\mathrm{~m}_{b}$ & Mass of flexible backbone & $0.0326 \mathrm{Kg}$ \\
$\mathrm{m}_{d}$ & Disk mass & $0.0082 \mathrm{Kg}$ \\
$d_{b}$ & Diameter of flexible backbone & $0.005 \mathrm{~m}$ \\
$d_{d}$ & Diameter of disk & $0.04 \mathrm{~m}$ \\
$r$ & Radial distance between the cables and the neutral axis & $0.019 \mathrm{~m}$ \\
$\mathrm{E}$ & Elasticity modulus & $9.5 \mathrm{GPa}$ \\
\hline \hline
\end{tabular}
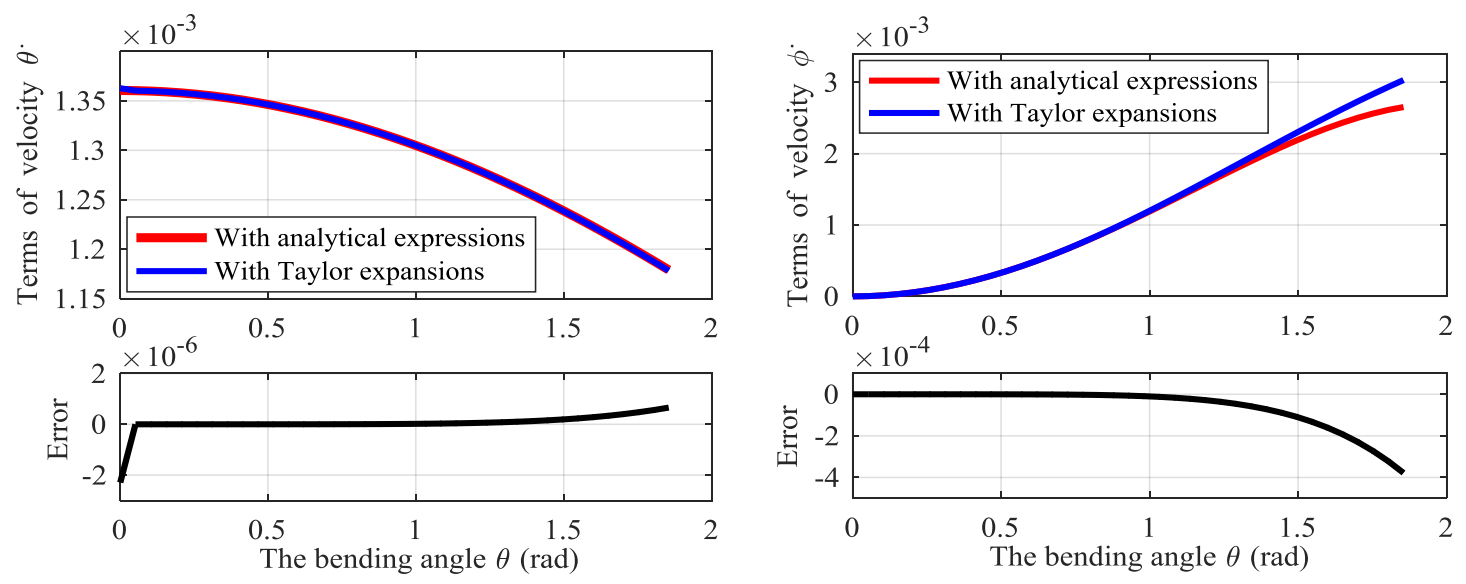

Fig. 6 The comparison of velocities' terms of the total kinetic energy, calculated by using exact expressions and those by Taylor expansions

From the analysis of Figs (2)-(5), it can be seen that the curves, of exact and equivalent factors, are much superposed except the factor $H_{8}$. Generally, CDCRs are lightweight and therefore, for the estimated parameters and geometric properties of 2-DOF CDCR under consideration are given in Table 1, the maximum error value between exact terms and that calculated by using equivalent factors of velocity terms of the total kinetic energyis less than $0.05 \%$ (precisely, this value is recorded at $\theta= \pm 3 \pi / 5$, see Fig. 6). This result indicates that the simplified expression of kinetic energy is satisfactory in the specified range of the bending angle.

\subsection{Total potential energy}

For the considered 2-DOF CDCR, the total potential energy is calculated as a sum of gravitational energies and elastic energy. Due to the low weight of disks and the flexible backbone mentioned earlier, the sum of gravitational energies can be 
ignored in comparison with elastic energy. Fig. 7 shows the ratio between the sum of gravitational energy of all disks and the flexible backbone over elastic energy calculated as a function of the bending angle $\theta$. From this Figure, it can be seen that the sum of gravitational energy are much smaller than the elastic energy where the maximum value of the ratio is less than $0.27 \%$. Therefore, the potential energy can be calculated as [29]

$$
\mathrm{U}=\frac{\mathrm{EI}_{b}}{2 \ell} \theta^{2}
$$

where $\mathrm{E}$ is the elasticity modulus, $\mathrm{I}_{b}$ is the second moment of cross-sectional area of flexible backbone.

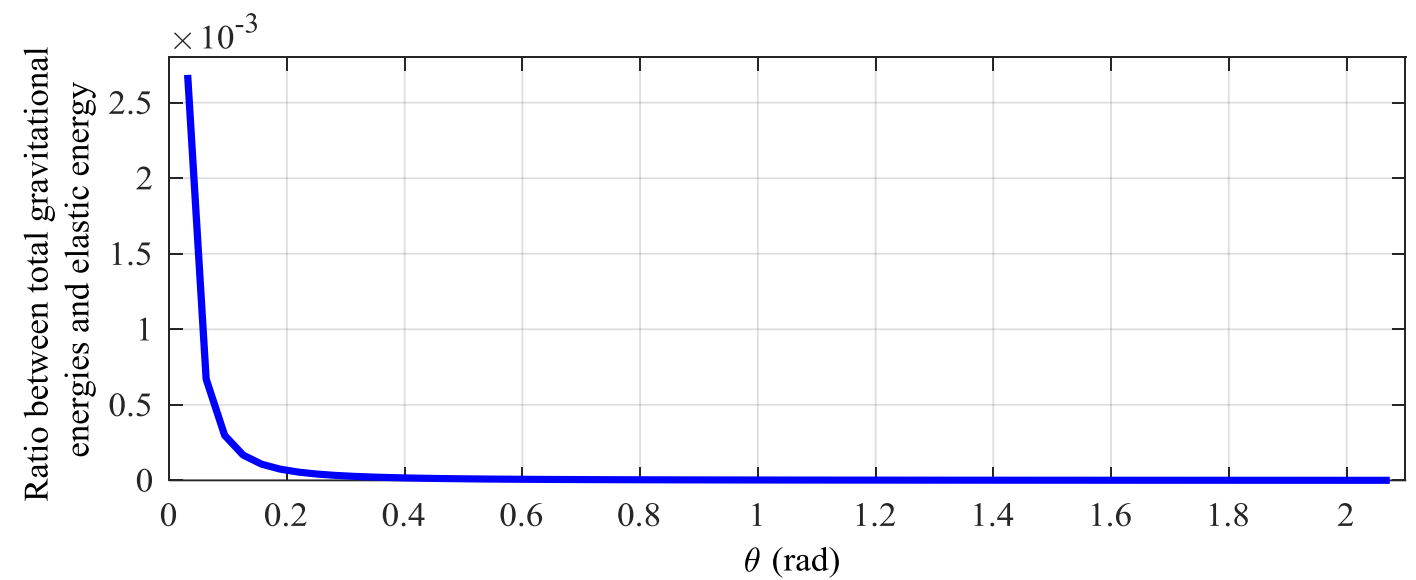

Fig. 7 Ratio between total gravitational energy and elastic energy as a function of bending angle

\subsection{Generalized forces}

As mentioned above that the CDCR under consideration has two degrees of freedom; thus, the spatial motion can be achieved by actuating one or two forces, at the same time, on the cables. So, the relationships between the generalized forces $\mathrm{Q}_{1}$ and $\mathrm{Q}_{2}$ in the range as $\varphi \in\left[\begin{array}{ll}0 & 2 \pi / 3\end{array}\right]$ can be expressed as a function of tension forces on the cables, $\mathrm{F}_{1}$ and $\mathrm{F}_{2}$, as [15]

$$
\left\{\begin{array}{l}
\mathrm{Q}_{1}=\mathrm{F}_{1} r \mathrm{c}\left(\gamma_{1}-\varphi\right)+\mathrm{F}_{2} r \mathrm{c}\left(\gamma_{2}-\varphi\right) \\
\mathrm{Q}_{2}=\mathrm{F}_{1} r \theta \mathrm{s}\left(\gamma_{1}-\varphi\right)+\mathrm{F}_{2} r \theta \mathrm{s}\left(\gamma_{2}-\varphi\right)
\end{array}\right.
$$

where $r$ is the distance from the central axis of flexible backbone to each actuating cable on the disk.

With all previously defined terms involved in dynamic model, the equations of motion will be derived in the following subsection.

\subsection{Equations of motion}

The resulted model from the application of Euler-Lagrange method can be written as

$$
\left[\begin{array}{ll}
M_{11} & M_{12} \\
M_{21} & M_{22}
\end{array}\right]\left\{\begin{array}{l}
\ddot{\theta} \\
\ddot{\varphi}
\end{array}\right\}+\left[\begin{array}{lll}
C_{11} & C_{12} & C_{13} \\
C_{21} & C_{22} & C_{23}
\end{array}\right]\left\{\begin{array}{l}
\dot{\theta}^{2} \\
\dot{\theta} \dot{\varphi} \\
\dot{\varphi}^{2}
\end{array}\right\}+\left[\begin{array}{cc}
K_{11} & K_{12} \\
K_{21} & K_{22}
\end{array}\right]\left\{\begin{array}{l}
\theta \\
\varphi
\end{array}\right\}=\left[\begin{array}{ll}
D_{11} & D_{12} \\
D_{21} & D_{22}
\end{array}\right]\left\{\begin{array}{l}
\mathrm{F}_{1} \\
\mathrm{~F}_{2}
\end{array}\right\}
$$

where

$$
\left\{\begin{array}{l}
M_{11}=\ell^{2} \mathrm{~m}_{b} H_{1}+\ell I_{b} H_{3}+\ell^{2} \mathrm{~m}_{d} H_{5}+\mathrm{I}_{x x} H_{7} \\
M_{12}=M_{21}=0 \\
M_{22}=\ell^{2} \mathrm{~m}_{b} H_{2}+\ell \mathrm{I}_{b} H_{4}+\ell^{2} \mathrm{~m}_{d} H_{6}+\mathrm{I}_{x x} H_{8}
\end{array}\right.
$$




$$
\begin{aligned}
& \left\{\begin{array}{l}
C_{11}=\frac{1}{2}\left(\ell^{2} \mathrm{~m}_{b} \frac{\partial H_{1}}{\partial \theta}+\ell \mathrm{I}_{b} \frac{\partial H_{3}}{\partial \theta}+\ell^{2} \mathrm{~m}_{d} \frac{\partial H_{5}}{\partial \theta}+\mathrm{I}_{x x} \frac{\partial H_{7}}{\partial \theta}\right) \\
C_{12}=C_{21}=C_{23}=0 \\
C_{13}=-\frac{1}{2}\left(\ell^{2} \mathrm{~m}_{b} \frac{\partial H_{2}}{\partial \theta}+\ell \mathrm{I}_{b} \frac{\partial H_{4}}{\partial \theta}+\ell^{2} \mathrm{~m}_{d} \frac{\partial H_{6}}{\partial \theta}+\mathrm{I}_{x x} \frac{\partial H_{8}}{\partial \theta}\right) \\
C_{22}=\left(\ell^{2} \mathrm{~m}_{b} \frac{\partial H_{2}}{\partial \theta}+\ell \mathrm{I}_{b} \frac{\partial H_{4}}{\partial \theta}+\ell^{2} \mathrm{~m}_{d} \frac{\partial H_{6}}{\partial \theta}+\mathrm{I}_{x x} \frac{\partial H_{8}}{\partial \theta}\right)
\end{array}\right. \\
& \begin{cases}K_{11}=\frac{\mathrm{EI}_{b}}{\ell} \\
K_{12}=K_{21}=K_{22}=0\end{cases} \\
& \begin{cases}D_{11}=r \mathrm{c}(\varphi) \\
D_{12}=r \mathrm{c}(2 \pi / 3-\varphi) \\
D_{21}=-r \theta \mathrm{s}(\varphi) \\
D_{22}=r \theta \mathrm{s}(2 \pi / 3-\varphi)\end{cases}
\end{aligned}
$$

\section{Simulation-Based Analysis}

The static equilibrium analysis, the forward dynamic responses and the inverse dynamic responses are successively presented.

\subsection{Static equilibrium analysis}

This example is carried out without tension in cables. The model is initialized with a value of the bending angle as $\theta=\pi / 4$ and a zero value of the orientation angle $(\varphi=0)$. The dynamic responses for two angles of the CDCR are shown in Fig. 8, where it can be seen that the robot presents oscillations around an equilibrium position (i.e. around the $Z_{0}$-axis). The stabilization of robot begins after $37.68 \mathrm{sec}$ with a sample step equal to $0.06 \mathrm{sec}$.

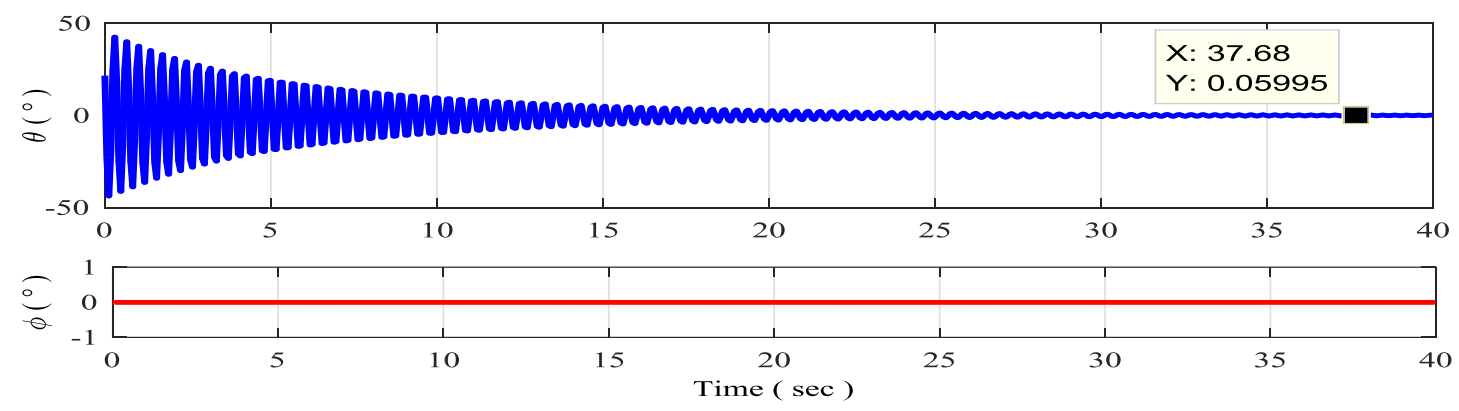

Fig. 8 Dynamic responses for the bending and the orientation angles with null actuation forces

\subsection{Forward dynamic responses (FDRs)}

This model is used to estimate the motion of the 2-DOF CDCR (i.e. the angles $\theta$ and $\varphi$ ) when the cables are actuated by tension forces. For this model, two simulations examples are performed. In Fig. 9, we present the FDRs for the bending and the orientation angles as a response to an input of $5 \mathrm{~N}$ of a tension in cable 1 from its equilibrium position. From this Figure, it can be seen that the continuum robot presents some oscillations around a new stable value of the bending angle which equals to $\theta=15.53^{\circ}$, while the orientation angle $\varphi$ remains constant and equals to zero.

In the second example, tensions are applied on cable 1 and 2 (see left side of Fig.1). By considering the tensions in the cables shown in Fig. 10(a), the FDRs for the Cartesian coordinates of the end-point of the robot are shown in Fig. 10(b). It is noticed that these coordinates are captured after stabilization of the dynamic model simulation. 

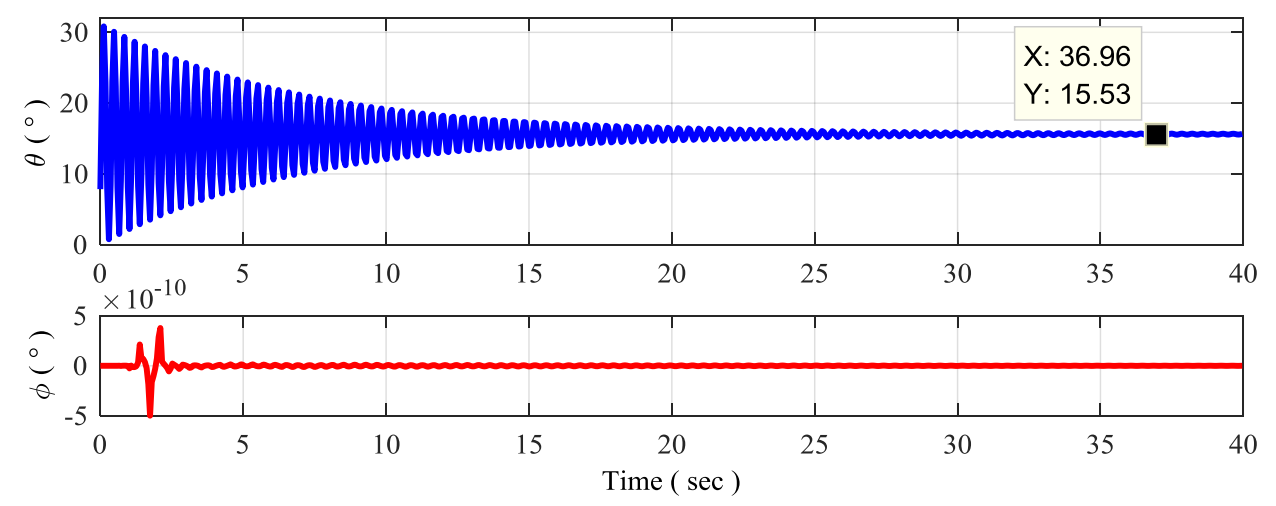

Fig. 9 FDRs for bending and orientation angles

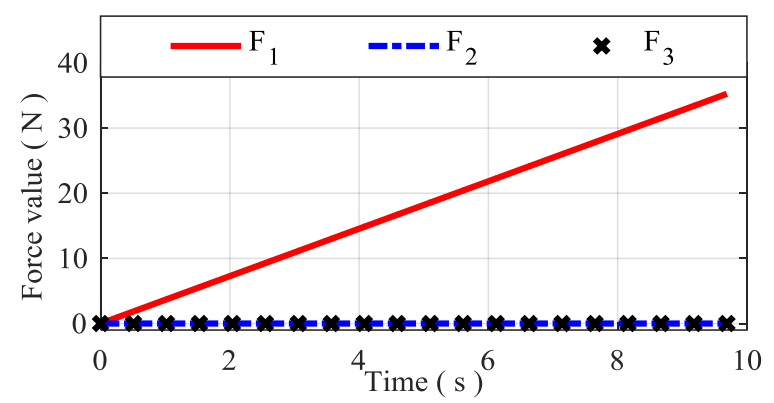

(a)Temporal evolution of cable tensions

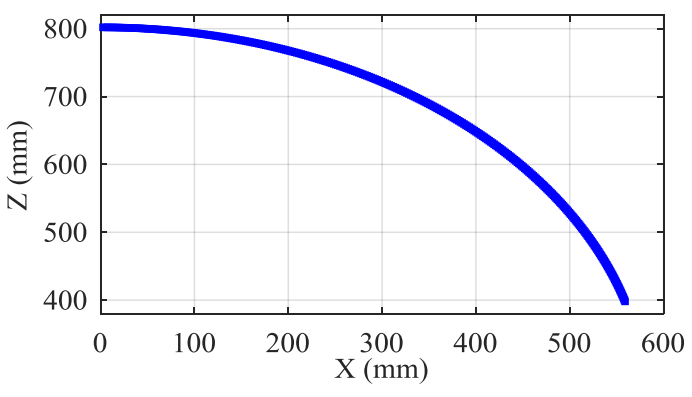

(b) Cartesian coordinates of the end-point of the robot

Fig. 10 Inputs and outputs of FDRs

\subsection{Inverse dynamic responses (IDRs)}

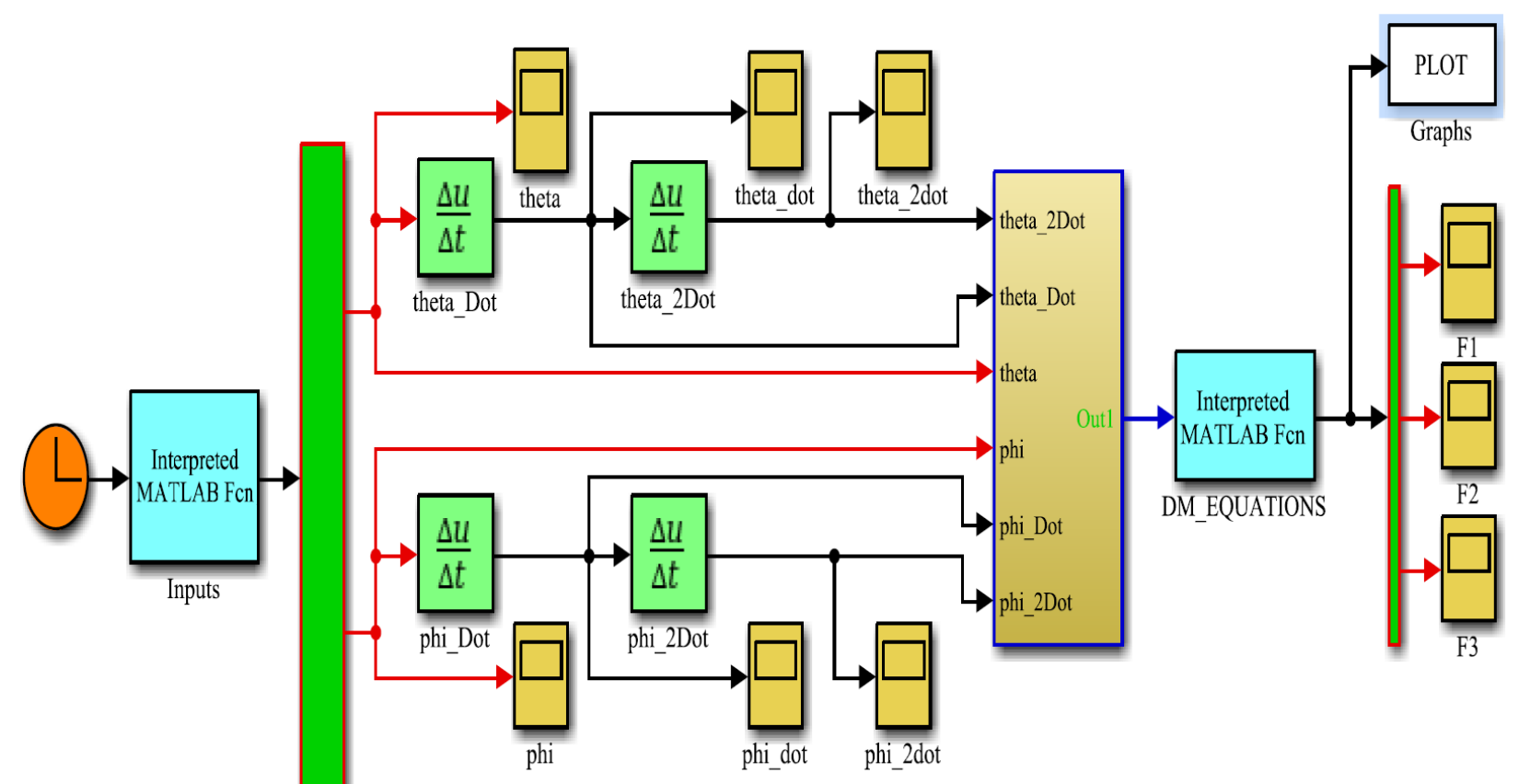

Fig. 11 2-DOF CDCR modeled in Matlab/Simulink

The dynamic model has been implemented in Matlab/Simulink including the numerical derivatives of velocities and accelerations, as shown in Fig. 11. The actuated forces on three cables required for the end-point of 2-DOF CDCR are estimated. Two simulation examples are considered. Fig. 12 illustrates the obtained simulation results from IDRs to track a spatial circular trajectory which is defined by $\theta=\frac{\pi}{12}$ and $\varphi=\frac{\pi}{5} t$ (red line), where $t$ is the sampling step of time varies from 0 to 10 with a step equal to 0.2 . 
In the second example, the obtained simulation results, shown in Fig. 13(b), represent the given data for tracking a trajectory defined by $\theta=\frac{\pi}{4} t$ and $\varphi=\frac{\pi}{6}$ (see Fig. 13(a)).

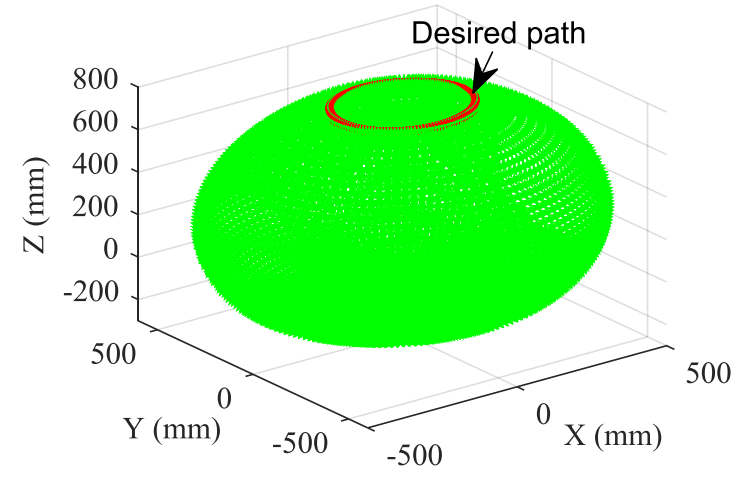

(a) Desired path plotted on 2-DOF CDCR's workspace

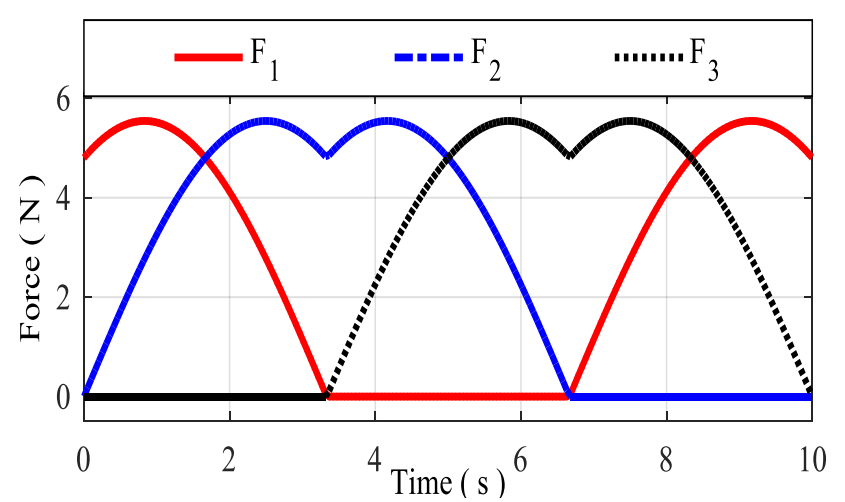

(b) Temporal evolution of the actuation forces

Fig. 12 Inputs and outputs of IDRs (Example 1)

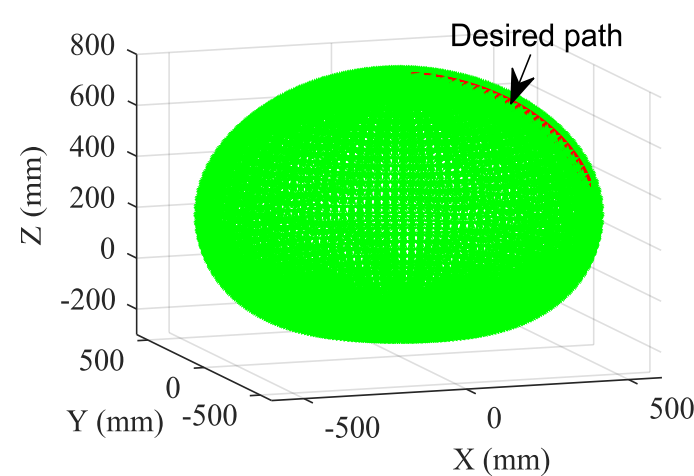

(a) Desired path plotted on 2-DOF CDCR's workspace

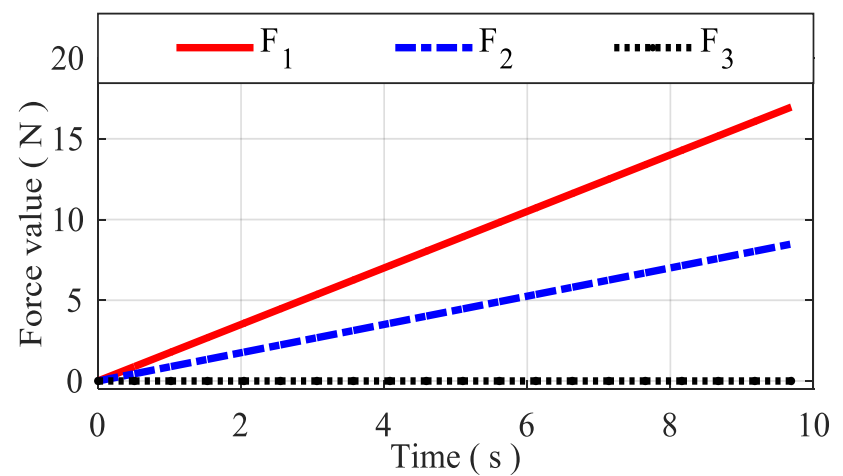

(b) Temporal evolution of the actuation forces

Fig. 13 Inputs and outputs of IDRs (Example 2)

\subsection{Dynamic responses with a PID controller}

In order to reduce the oscillations around the new stable value of the bending angle, as shown in Fig. 9, a Proportional-Integral-Derivative (PID) controller is integrated. For this, the resulting value of the FDRs of example 1 is used as an input for the proposed PID controller. The selected parameters of the PID controller offer an acceptable compromise on performance and their values are: $K_{P}=2.8, K_{I}=0.004$, and $K_{D}=0.38$.
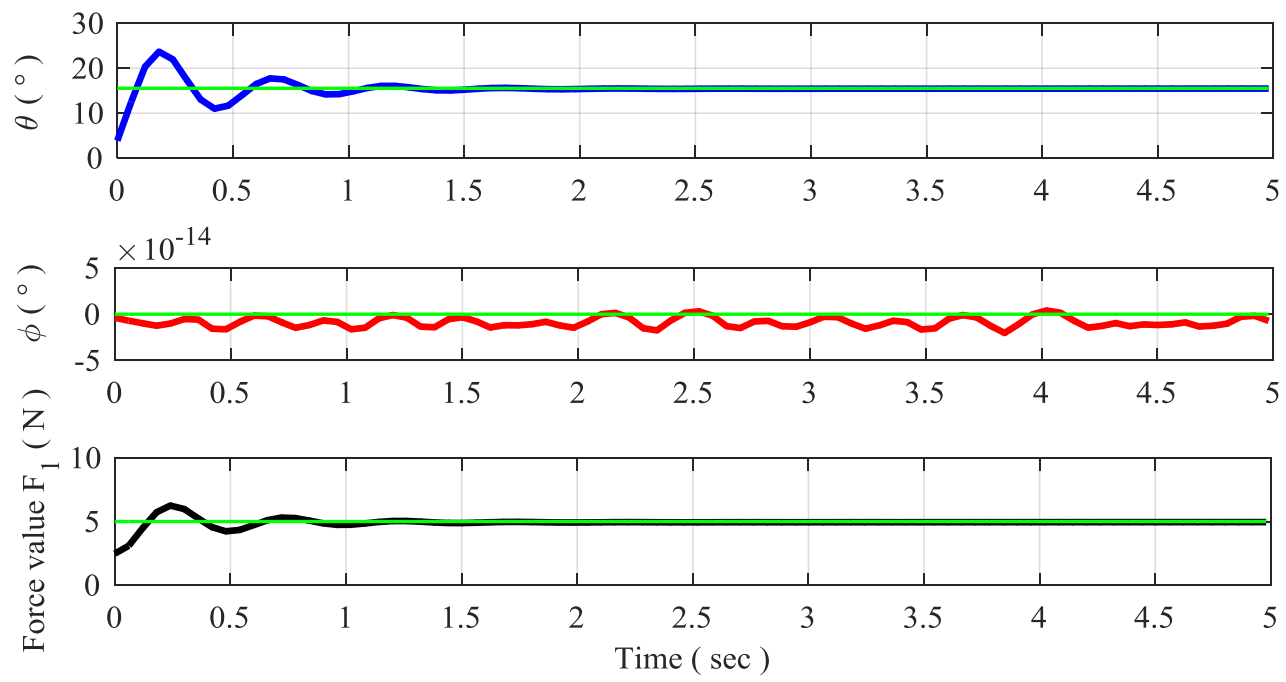

Fig. 14 Dynamic responses for the bending and orientation angles with the PID controller 
The dynamic responses in closed-loop with the PID controller are shown in Fig. 14. The observation of the graphical results illustrated in Figs. 9 and 14, shows that the classical PID controller is capable of reducing those oscillations significantly. The simulation results demonstrate that the PID controller is effective and suitable when it comes to improving the performance responses.

\subsection{Summary}

Generally, CDCRs have different characteristics and specificities such as size, weight, flexible backbone material, etc. Therefore, it is difficult to make a direct comparison among the proposed models only on the basis of the obtained results. Despite the differences in assumptions and methods used as well as the properties of studied CDCRs, it is deduced that the proposed dynamic model is almost similar in comparison to results of existing works [15, 25].

Considering the results on static equilibrium and despite some mentioned differences above, it can be seen that there is a very similar shape and behavior responses compared to work [25]. The same conclusion can be observed for forward dynamic models regardless of the inclusion of friction effects.

Considering the inverse dynamic model, to our best knowledge only the work [15] handles this model. Therefore, the same path trajectory presented in the paper [15], was used to validate our proposed model. From the analysis of the obtained results, it can be seen that there is a great similarity from the point of view of output responses.

On the other hand, comparing the present model to the one developed in the previous work [27], it is found that the obtained expressions are simple. The maximum error value between exact terms of the velocities of the total kinetic energy and ones calculated by using the equivalent factors is less than $0.05 \%$ in the specified range of the bending angle.

As a general conclusion of this analysis, since there are some similarities between the obtained results for the static and dynamic models compared to available works; this enables to validate the proposed dynamic model and therefore to adopt this model for research studies as well as for control implementation purposes. These topics will be considered for future research projects.

\section{Conclusions}

In this paper, the dynamic model of 2-DOF CDCR was established based on Taylor expansions approximations through the Euler-Lagrange method. The approach extends the previous work [27] by modeling the three dimensional case taking into account other parameters such as the inertial terms. To this end, some simplifications have been adopted to reduce the dynamic model's complexity. The obtained expressions of the dynamic model are relatively simple which enabled an easier analysis and simulation of the CDCR behavior. Specifically, the simplified expressions of velocity's terms of the total kinetic energy are provided with a maximum error value less than $0.05 \%$ in the specified range of the bending angle of the robot. On the other hand, due to the low weight of CDCR components, the total gravitational energy was neglected relative to the elastic energy of the flexible backbone. The estimated maximum ratio is less than $0.27 \%$. The obtained simulation results for both static and dynamic models are being more or less similar compared to the available literature works, despite differences on assumptions, modeling approaches and specificities of the considered CDCR. However, more bending sections increase the complexity of the dynamic model despite the used simplifications. Actually, the obtained dynamic model can be exploited to test and implement control purposes.

\section{Conflicts of Interest}

The authors declare no conflict of interest. 


\section{Nomenclature}

\begin{tabular}{|l|l|}
\hline$d_{b}$ & Diameter of the flexible backbone \\
\hline$d_{b}$ & Diameter of the disk \\
\hline $\mathrm{E}$ & Elasticity modulus \\
\hline $\mathrm{F}_{i}$ & Tension force in the cable $i$ \\
\hline$i$ & Index of the cable, $i=1,2,3$ \\
\hline $\mathrm{I}$ & Disk's moment of inertia expressed in the frame $\left\{X_{0}, Y_{0}, Z_{0}\right\}$ \\
\hline $\mathrm{I}_{b}$ & Inertia moment of the flexible backbone \\
\hline $\mathrm{I}_{d}$ & Inertia moment of the disk \\
\hline $\mathrm{I}_{x x}, \mathrm{I}_{y y}, \mathrm{I}_{z z}$ & Disk's moment of inertia aligned with $X, Y$ and $Z$ axis, respectively \\
\hline$k$ & Index of the disk, $k=1,2, \ldots, 10$ \\
\hline$\ell$ & Length of the flexible backbone \\
\hline $\mathrm{m}_{b}$ & Mass of the flexible backbone \\
\hline $\mathrm{m}_{d}$ & Mass of the disk \\
\hline $\mathrm{n}_{s}, \mathrm{~b}_{s}, \mathrm{t}_{s}$ & Unit vectors of matrix $\mathrm{R}_{s}$ \\
\hline $\mathrm{Q}_{j}$ & Generalized forces, $j=1,2$ \\
\hline$r$ & Radial distance between the cables and the neutral axis of the flexible backbone \\
\hline $\mathrm{r}_{s}$ & Position vector \\
\hline $\mathrm{R}_{s}$ & Rotation matrix \\
\hline$s$ & Curve parameter \\
\hline$t$ & Time variable \\
\hline $\mathrm{T}$ & Total kinetic energy \\
\hline $\mathrm{U}$ & Total potential energy \\
\hline $\mathrm{v}_{s}$ & Linear velocity \\
\hline$\kappa$ & Curvature \\
\hline$\varphi$ & Orientation angle \\
\hline$\theta$ & Bending angle \\
\hline$\omega_{s}$ & Angular velocity \\
\hline$\cdot$ & Denoted first derivative with respect to time \\
\hline$\wedge$ & Denoted skew matrix \\
\hline & \\
\hline & \\
\hline & \\
\hline & \\
\hline
\end{tabular}

\section{Appendix}

$$
\begin{aligned}
H_{3}= & \frac{5}{8}-\frac{15}{64 \theta^{2}}+\frac{\mathrm{c}^{2}(\theta)}{2 \theta^{2}}-\frac{\mathrm{s}(2 \theta)}{8 \theta^{3}}-\frac{\mathrm{s}(4 \theta)}{256 \theta^{3}} \\
H_{4}= & \frac{1}{2}-\frac{\theta \mathrm{s}(2 \theta)}{4 \theta^{2}} \\
H_{5}= & \frac{1}{40 \theta^{4}}\left(400-40 \mathrm{c}\left(\frac{\theta}{2}\right)-40 \mathrm{c}\left(\frac{\theta}{5}\right)-40 \mathrm{c}\left(\frac{2 \theta}{5}\right)-40 \mathrm{c}\left(\frac{3 \theta}{5}\right)-40 \mathrm{c}\left(\frac{4 \theta}{5}\right)-40 \mathrm{c}\left(\frac{\theta}{10}\right)-40 \mathrm{c}\left(\frac{3 \theta}{10}\right)-40 \mathrm{c}\left(\frac{7 \theta}{10}\right)\right. \\
& -40 \mathrm{c}\left(\frac{9 \theta}{10}\right)+77 \theta^{2}-40 \mathrm{c}(\theta)-40 \theta \mathrm{s}(\theta)-20 \theta \mathrm{s}\left(\frac{\theta}{2}\right)-8 \theta \mathrm{s}\left(\frac{\theta}{5}\right)-16 \theta \mathrm{s}\left(\frac{2 \theta}{5}\right)-24 \theta \mathrm{s}\left(\frac{3 \theta}{5}\right)-32 \theta \mathrm{s}\left(\frac{4 \theta}{5}\right) \\
& \left.-4 \theta \mathrm{s}\left(\frac{\theta}{10}\right)-12 \theta \mathrm{s}\left(\frac{3 \theta}{10}\right)-28 \theta \mathrm{s}\left(\frac{7 \theta}{10}\right)-36 \theta \mathrm{s}\left(\frac{9 \theta}{10}\right)\right)
\end{aligned}
$$




$$
\begin{aligned}
H_{6}= & \frac{1}{4 \theta^{2}}\left(30+\mathrm{c}(2 \theta)-4 \mathrm{c}\left(\frac{\theta}{2}\right)-3 \mathrm{c}\left(\frac{\theta}{5}\right)-3 \mathrm{c}\left(\frac{2 \theta}{5}\right)-3 \mathrm{c}\left(\frac{3 \theta}{5}\right)-3 \mathrm{c}\left(\frac{4 \theta}{5}\right)-4 \mathrm{c}\left(\frac{\theta}{10}\right)+\mathrm{c}\left(\frac{6 \theta}{5}\right)+\mathrm{c}\left(\frac{7 \theta}{5}\right)-4 \mathrm{c}\left(\frac{3 \theta}{10}\right)\right. \\
& \left.+\mathrm{c}\left(\frac{8 \theta}{5}\right)+\left(\frac{9 \theta}{5}\right)-4 \mathrm{c}\left(\frac{7 \theta}{10}\right)-4 \mathrm{c}\left(\frac{9 \theta}{10}\right)-3 \mathrm{c}(\theta)\right) \\
H_{7}= & \frac{1}{32} \mathrm{c}(2 \theta)-\frac{99}{200} \mathrm{c}\left(\frac{\theta}{5}\right)-\frac{303}{800} \mathrm{c}\left(\frac{2 \theta}{5}\right)-\frac{91}{200} \mathrm{c}\left(\frac{3 \theta}{5}\right)-\frac{17}{50} \mathrm{c}\left(\frac{4 \theta}{5}\right)-\frac{207}{800} \mathrm{c}\left(\frac{6 \theta}{5}\right)-\frac{51}{200} \mathrm{c}\left(\frac{7 \theta}{5}\right)-\frac{27}{200} \mathrm{c}\left(\frac{8 \theta}{5}\right) \\
& -\frac{19}{200} \mathrm{c}\left(\frac{9 \theta}{5}\right)+\frac{1}{50} \mathrm{c}\left(\frac{12 \theta}{5}\right)+\frac{9}{800} \mathrm{c}\left(\frac{14 \theta}{5}\right)+\frac{1}{200} \mathrm{c}\left(\frac{16 \theta}{5}\right)+\frac{1}{800} \mathrm{c}\left(\frac{18 \theta}{5}\right)-\frac{3}{8} \mathrm{c}(\theta)+\frac{1051}{160} \\
H_{8}= & \mathrm{s}^{2}\left(\frac{\theta}{2}\right)+\mathrm{s}^{2}\left(\frac{\theta}{5}\right)+\mathrm{s}^{2}\left(\frac{2 \theta}{5}\right)+\mathrm{s}^{2}\left(\frac{3 \theta}{5}\right)+\mathrm{s}^{2}\left(\frac{4 \theta}{5}\right)+\mathrm{s}^{2}\left(\frac{\theta}{10}\right)+\mathrm{s}^{2}\left(\frac{3 \theta}{10}\right)+\mathrm{s}^{2}\left(\frac{7 \theta}{10}\right)+\mathrm{s}^{2}\left(\frac{9 \theta}{10}\right)+\mathrm{s}^{2}(\theta)
\end{aligned}
$$

\section{References}

[1] L. Fryziel, "Modélisation et calibrage pour la commande d'un micro-robot continuum dédié à la chirurgie mini-invasive," Ph.D. dissertation, Université Paris Est Créteil Val de Marne, France, December 2010.

[2] D. Trivedi, C. D. Rahn, W. M. Kier, and I. D. Walker, "Soft robotics: biological inspiration, state of the art, and future research," Applied Bionics and Biomechanics, vol. 5, no. 3, pp. 99-117, 2008.

[3] G. Robinson and J. B. C. Davies, "Continuum robots - a state of the art," Proc.IEEE International Conference on Robotics and Automation, Detroit, Michigan, May 1999, pp. 2849-2854.

[4] G. S. Chirikjian and J. W. Burdick, “A modal approach to hyper-redundant manipulator kinematics,” IEEE Transactions on Robotics and Automation, vol. 10, no. 3, pp. 343-354, June 1994.

[5] T. M. Bieze, "Contribution to kinematic modeling and control of soft manipulators using computational mechanics," Ph.D. dissertation, Université des Sciences et Technologies de Lille, France, October 2017.

[6] R. Cieslak and A.Morecki, "Elephant trunk type elastic manipulator a tool for bulk and liquid type materials transportation,” Robotica, vol. 17, no. 1, pp. 11-16, January 1999.

[7] I. D. Walker and M. W. Hannan, “A novel elephant's trunk' robot," Proc. IEEE/ASME International Conference on Advanced Intelligent Mechatronics, September 1999, pp. 410-415.

[8] O. Lakhal, "Contribution to the modeling and control of hyper-redundant robots: application to additive manufacturing in the construction," Ph.D. dissertation, Université des Sciences etTechnologies de Lille, France, January 2019.

[9] W. McMahan, B. A. Jones, and I. D. Walker, "Design and implementation of a multi-section continuum robot: Air-Octor," Proc. IEEE International Conference on Intelligent Robots and Systems, August 2005, pp. 2578-2585.

[10] W. McMahan and I. D. Walker, "Octopus-inspired grasp-synergies for continuum manipulators," Proc. IEEE International Conference on Robotics and Biomimetics, February 2009, pp.945-950.

[11] S. Mosqueda, Y. Moncada, C. Murrugarra, and H. Leon-Rodriguez, "Constant curvature kinematic model analysis and experimental validation for tendon driven continuum manipulators," Proc. the 15th International Conference on Informatics in Control, Automation and Robotics (ICINCO 2018), vol. 2, 2018, pp.211-218.

[12] Y. Tian, M. Luan, X. Gao, W. Wang, and L. Li, "Kinematic analysis of continuum robot consisted of driven flexible rods," Mathematical Problems in Engineering, vol. 2016, pp. 1-7, 2016.

[13] J. Tang, Y. Zhang, F. Huang, J. Li, Z. Chen, W. Song, S. Zhu, and J. Gu, "Design and kinematic control of the cable-driven hyper-redundant manipulator for potentialunderwater applications," Applied Sciences, vol. 9, no. 6, pp. 1-20, 2019.

[14] A. K. Mishra, A. Mondini, E. Del Dottore, A. Sadeghi, F. Tramacere, and B. Mazzolai, "Modular continuum manipulator: Analysis and characterization of its basic module," Biomimetics, vol. 3, no. 1, pp. 1-16, 2018.

[15] B. He, Z. Wang, Q. Li, H. Xie, and R. Shen, “An analytic method for the kinematics and dynamics of a multiple-backbone continuum robot," International Journal of Advanced Robotic Systems, vol. 10, pp. 1-13, 2013.

[16] A. Amouri, C. Mahfoudi, A. Zaatri, O. Lakhal, and R. Merzouki, "A metaheuristic approach to solve inverse kinematics of continuum manipulators," Journal of systems and Control Engineering, vol. 231, no. 5, pp. 380-394, 2017.

[17] H. El-Hussieny, S. G. Jeong, and J. H. Ryu, "Dynamic modeling of a class of soft growing robots using Euler-Lagrange formalism," Proc. the SICE Annual Conference, Society of Instrument and Control Engineers (SICE), Hiroshima, Japan, September 10-13, 2019.

[18] R. J. Webster and B. A. Jones, "Design and kinematic modeling of constant curvature continuum robots: A review," International Journal of Robotics Research, vol. 29, no. 13, pp. 1661-1683, 2010. 
[19] V. Falkenhahn, T Mahl, A. Hildebrandt, A. Hildebrandt, R. Neumann, and O. Sawodny, "Dynamic modeling of bellows-actuated continuum robots using the Euler-Lagrange formalism," IEEE Transactions on Robotics, vol. 31, no. 6, pp. 1483-1496, December 2015.

[20] D. C. Rucker and R. J. Webster III, "Statics and dynamics of continuum robots with general tendon routing and external loading," IEEE Transactions on Robotics, vol.27, no. 6, pp. 1033-1044, July 2011.

[21] A. Abu Alqumsan, S. Khoo, and M. Norton, "Robust control of continuum robots using Cosserat rod theory," Mechanism and Machine Theory, vol. 131, pp. 48-61, January 2019.

[22] F. Renda, F. Boyer, J. Dias, and L.Seneviratne, "Discrete Cosserat approach for multisection soft manipulator dynamics," IEEE Transactions on Robotics, vol. 34, no. 6, 2018.

[23] F. Matsuno and H. Sato, "Trajectory tracking control of snake robots based on dynamic model," In: Proc. IEEE International Conference of Robotics and Automation, Barcelona, Spain, April 2005, pp. 3040-3045.

[24] A. Gravagne, C. D. Rahn, and I. D. Walker, "Large deflection dynamics and control for planar continuum robots," IEEE/ASME Transactions on Mechatronics, vol. 8, no. 2, pp. 299-307, 2003.

[25] W. Rone and P.Ben-Tzvi, "Continuum robot dynamics utilizing the principle of virtual power," IEEE Transactions on Robotics, vol. 30, no. 1, pp. 275-287, February 2014.

[26] W. Rone and P. Ben-Tzvi, "Mechanics modeling of multi-segment rod-driven continuum robots," Journal of Mechanisms and Robotics," vol. 6, no. 4, November 2014.

[27] A. Amouri, A. Zaatri, and C. Mahfoudi, "Dynamic modeling of a class of continuum manipulators in fixed orientation," Journal of Intelligent and Robotic Systems, vol. 91, no. 3-4, pp. 413-424, 2018.

[28] M. Dehghani and S. Ali A.Moosavian, "Dynamics modeling of a continuum robotic arm with a contact point in planar grasp," Journal of Robotics, vol. 2014, Article ID 308283, 2014.

[29] D. G. Fertis, “Advanced mechanics of structure,” Marcel Dekker, Inc., New York City, 1996.

Copyright $\odot$ by the authors. Licensee TAETI, Taiwan. This article is an open access article distributed under the terms and conditions of the Creative Commons Attribution (CC BY-NC) license (https://creativecommons.org/licenses/by-nc/4.0/). 